\title{
DESIGN OF HIGH GAIN ANTENNA BASED ON ARRAY OF DOUBLE SLOT VIVALDI STRUCTURE
}

\section{*Shams Khaled Ahmed ${ }^{1}$}

1) Electrical Engineering, College of Engineering, Mustansiriyah University Baghdad, Iraq

2) Electrical Engineering, College of Engineering, Mustansiriyah University Baghdad, Iraq

\section{Zaid A. Abdul Hassain²}

\begin{abstract}
The classical Vivaldi antenna is recognized for its ultra-wideband property, however average gain and low directivity. To enhance the directivity, a Double slot construction with $120 \times 80 \times 1 \mathrm{~mm}^{3}$ size has been proposed. A T-junction power divider with a steady amplitude and phase is utilized to provide excitation for the two slots. The Double slot construction has the ability to create standard-like waves in the E-plane of the antenna. As an effect, the gain of the double slot Vivaldi antenna is greatly enhanced matched to the classical Vivaldi antenna. The consequences present that the frequency domain of the antenna is $(3-10) \mathrm{GHz}$. also an elliptical lens has been added to furthermore improve the Gain of the designed antenna to $14.5 \mathrm{~dB}$.
\end{abstract}

Keywords: Vivaldi antenna, double slot, high gain, ultrawideband, bandwidth, directivity

\section{Introduction}

The classical Vivaldi Antenna is a travelingwave end-fire standard antenna display wide bandwidth, medium gain [1-2], and the main beam divides in small multi beams when the VA is stamped on thick dielectric substrates [3-5]. Several methods like, utilizing directors at the opening of the antenna [6], and employing a dielectric lens [7] To enhance the gain, also an array of VA [9] [8] is proposed as a classic method to acquire elevated directivity, however, it is complicated, bulky, and costly for some applications [9-16]. For the classical VA array, the minimal electrical space among the elements should be larger than $\lambda / 2$. Otherwise to achieve a decrease in the spacing among the array elements a Double slot construction is proposed. In this paper, a Double slot Vivaldi antenna (DSVA) is simulated and optimizes established by (HFSS) Ansoft's High-Frequency Structure Simulator [17].

\section{Double Slot Vivaldi Antenna Design}

The proposed DSVA configuration is given in Fig.1. The antenna is stamped on the FR4 substrate of a $120 \times 80 \times 1 \mathrm{~mm}^{3}$ size and relative dielectric constant $\varepsilon_{\mathrm{r}}=4.4$. The DSVA formed from two exponential tapered slot and Microstrip slot-line transitions are utilized to feed slots of the antenna. The DSVA be operated in a steady amplitude and phase by utilizing a T-junction power divider. An exponential taper is utilized for the DSVA to transform $100 \Omega$ to $50 \Omega$. The double-slot construction may create standard-like waves in the E-plane of the antenna.

*Corresponding Author: memememe.19961993@gmail.com 


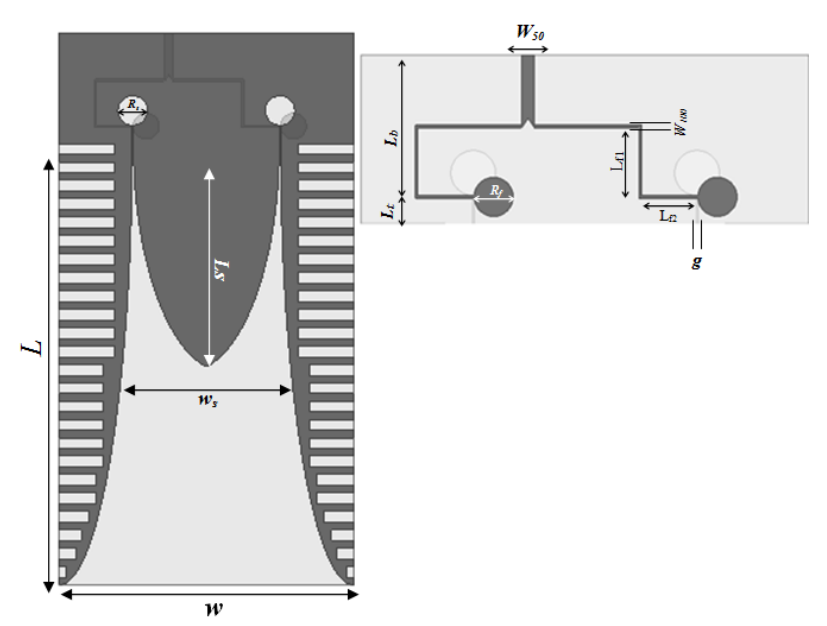

Figure1. DSVA configuration

The structural parameters of the DSVA are listed in the following Table 1 in $\mathrm{mm}$ which has been optimized:

Table1. DSVA dimensions in $\mathrm{mm}$.

\begin{tabular}{ccccccc}
\hline $\mathrm{W}$ & $\mathrm{L}$ & $\mathrm{L}_{\mathrm{b}}$ & $\mathrm{g}$ & $\mathrm{L}_{\mathrm{s}}$ & $\mathrm{W}_{\mathrm{s}}$ & $\mathrm{L}_{\mathrm{f} 1}$ \\
80 & 120 & 25 & 0.3 & 60 & 40 & 10 \\
\hline $\mathrm{L}_{\mathrm{t}}$ & $\mathrm{R}_{\mathrm{f}}$ & $\mathrm{Rs}$ & $\mathrm{L}_{\mathrm{f} 2}$ & $\mathrm{~W}_{100}$ & $\mathrm{~W}_{50}$ & \\
5 & 3.5 & 4 & 12 & 0.5 & 0.3 & \\
\hline
\end{tabular}

The DSVA consists of four exponential tapered curves [45]:

$$
\begin{aligned}
& \mathrm{E}_{1}: \mathrm{y}=\frac{1}{2} \times\left(\mathrm{W}_{\mathrm{s}}-\mathrm{g} \times \exp \left(\ln \left(\frac{\mathrm{W}_{\mathrm{s}}}{\mathrm{g}}\right) \times \frac{\mathrm{x}}{\mathrm{L}_{\mathrm{s}}}\right)\right)(1) \\
& 0 \leq \mathrm{x} \leq \mathrm{L}_{\mathrm{s}} \\
& \mathrm{E}_{2}: \mathrm{y}=\frac{1}{2} \times\left(\mathrm{W}_{\mathrm{s}}+\mathrm{g} \times \exp \left(\ln \left(\frac{\mathrm{W}-\mathrm{W}_{\mathrm{s}}}{\mathrm{g}}\right) \times \frac{\mathrm{x}}{\mathrm{L}}\right)\right)(2) \\
& 0 \leq \mathrm{x} \leq \mathrm{L}
\end{aligned}
$$

The DSVA edge will be corrugated by seven gropes of deferent length rectangular slots that enhance the reflection coefficient, the gain, and the directivity. By using the slotted edges, the track of the surface current is expanded. Further, the slotted edges make the superficial current nearby the tapered slot which concentrates the energy. The slots width is $3 \mathrm{~mm}$ and the length of the slots gropes are $(2,4.5,6,8,10,12$, and 15) mm sequentially.

The proposed DSVA operational frequency domain is from $(3 \mathrm{GHz})$ to more than $(10 \mathrm{GHz})$. The characteristic properties of the designed antenna will be summarized and briefly demonstrated.

\subsection{Reflection Coefficient Calculation}

The variation of the reflection coefficient $S_{11}$ with frequency is presented in Fig. 2 for the proposed slotted edges DSVA and the DSVA without slots. It is noticed that the slots on the edges minimize $S_{11}$ to less than $(-10) \mathrm{dB}$ for the domain from (3 -12) GHz.

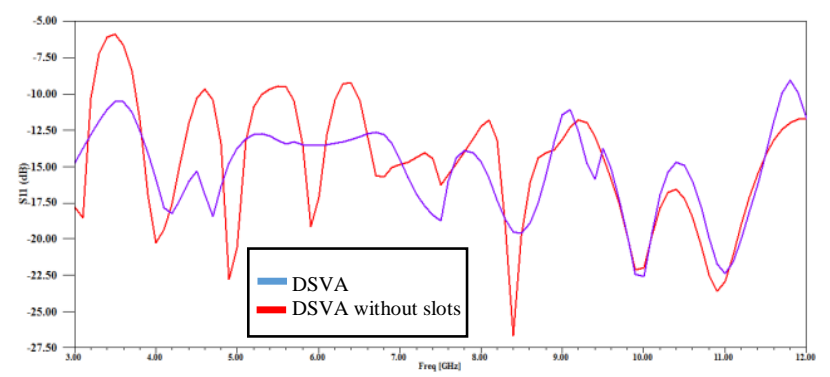

Figure2. Variation of $S_{11}$ with the frequency

\subsection{Radiation Patterns}

The gain pattern of the DSVA is calculated and plotted in 3D-form for three frequencies (3.1 $\mathrm{GHz}, 6.77 \mathrm{GHz}$, and $10 \mathrm{GHz}$ ) as shown in Fig.3.

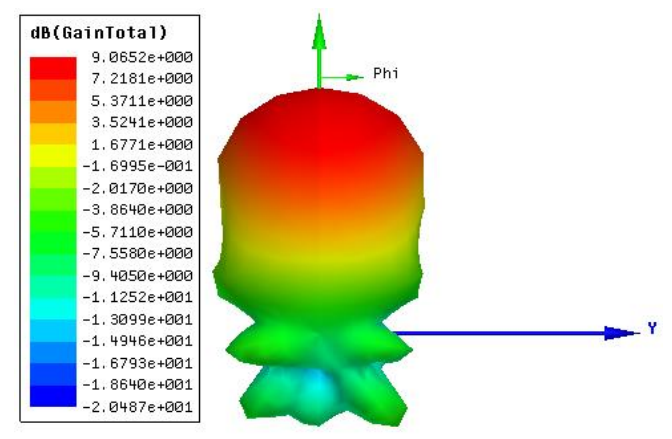

(a) 


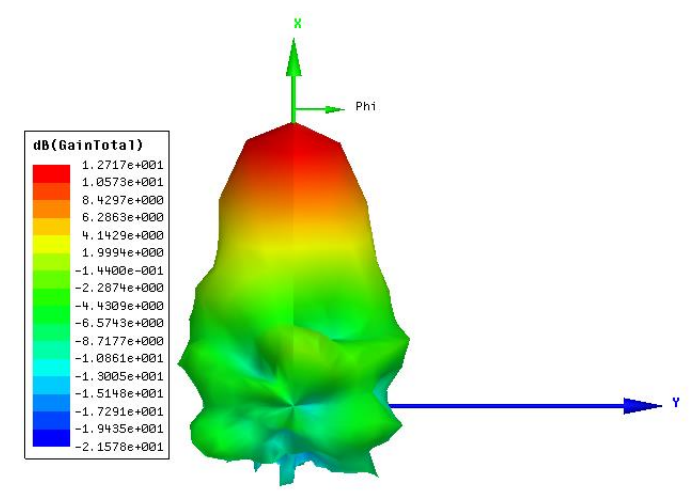

(b)

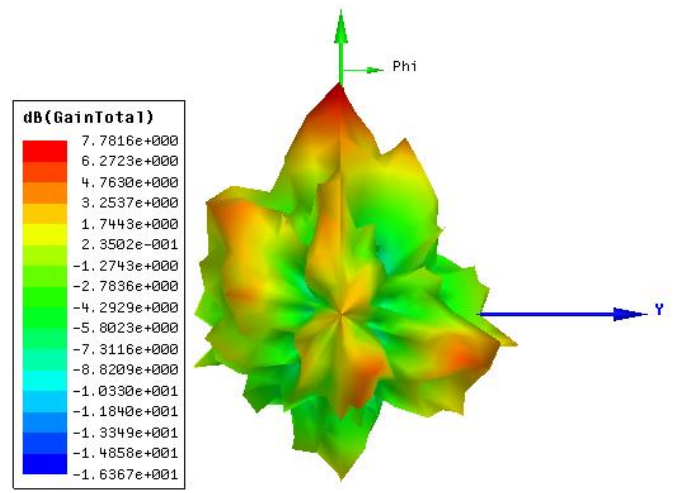

(c)

Figure3. Radiation patterns: (a) at $3.1 \mathrm{GHz}$, (b) at $6.77 \mathrm{GHz}$, and (c) at $10 \mathrm{GHz}$.

The realized gain of the DSVA variation is shown in Fig.4. It may be noticed that the ultimate gain obtained from the DSVA is $13 \mathrm{~dB}$.

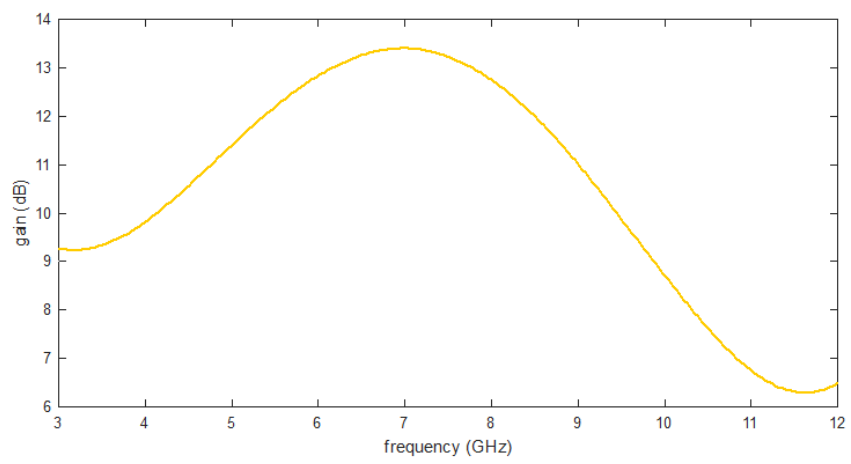

Figure4. Gain variation with frequency.
3. Modified DSVA Based on Dielectric Lens

To enhance the gain and directivity furthermore, a lens has been inserted into the structure. Proper lens shape is necessary to provide effective focusing in the end-fire direction. A half elliptical shape dielectric lens is proposed. The dielectric lens is achieved by extending the substrate to form the lens. The half elliptic fashion dielectric lens concentrates energy toward the middle of the opening of the DSVA. The designed DSVA with dielectric lens $(\mathrm{Ln}=60 \mathrm{~mm})$ is shown in Fig.5.

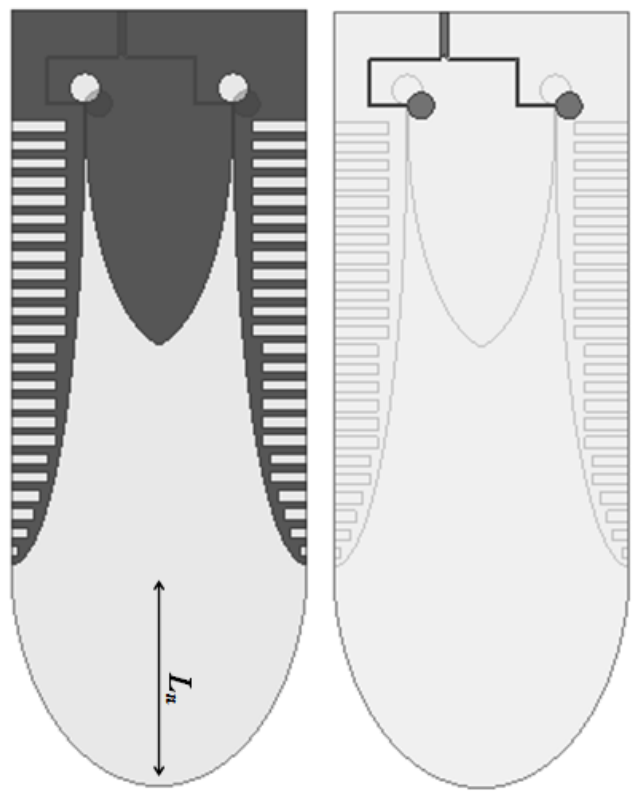

Figure5. DSVA with lens configuration

\subsection{Reflection Coefficient Calculation}

The variations of $S_{11}$ with frequency for both the DSVA and the modified DSVA with the lens are shown in Fig.6. It is noticed that $S_{11}$ is less than (-10) $\mathrm{dB}$ for the frequency domain (3-12) $\mathrm{GHz}$ which similar to the DSVA. 


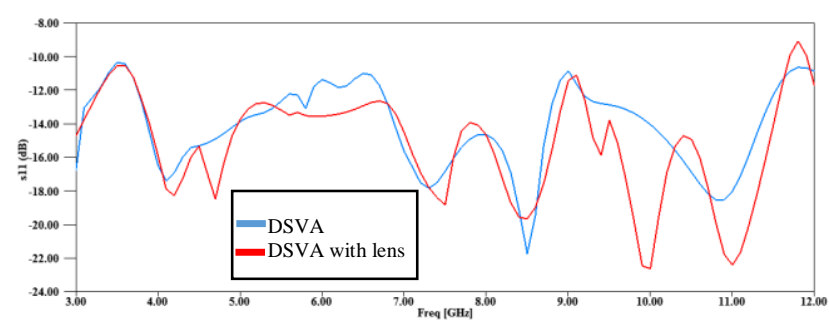

Figure6. Variation of $\mathrm{S}_{11}$ with the frequency of DSVA with the lens

\subsection{Radiation Patterns}

The gain pattern of the DSVA with the lens is calculated and plotted in 3D-form for three frequencies (3.1, 6.77, and 10) $\mathrm{GHz}$ as presented in Fig.7. It is noticed that the corrugated DSVA with the lens shows a steady end-fire radiation pattern in the low and center frequency.
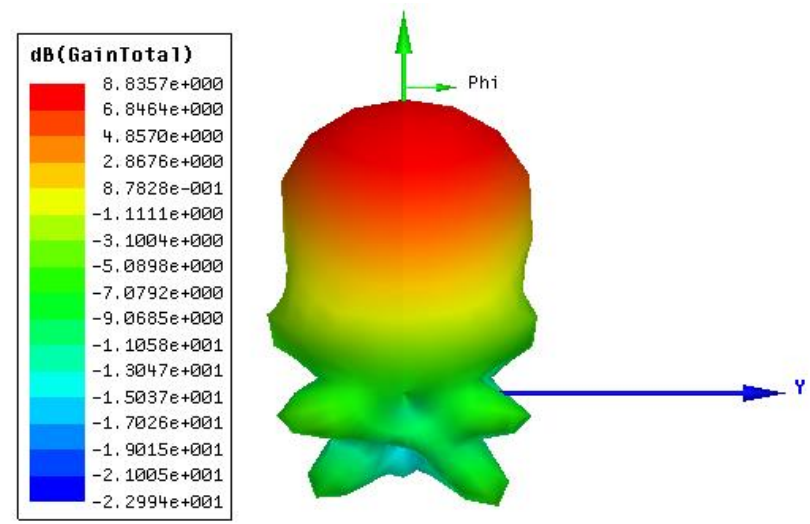

(a)
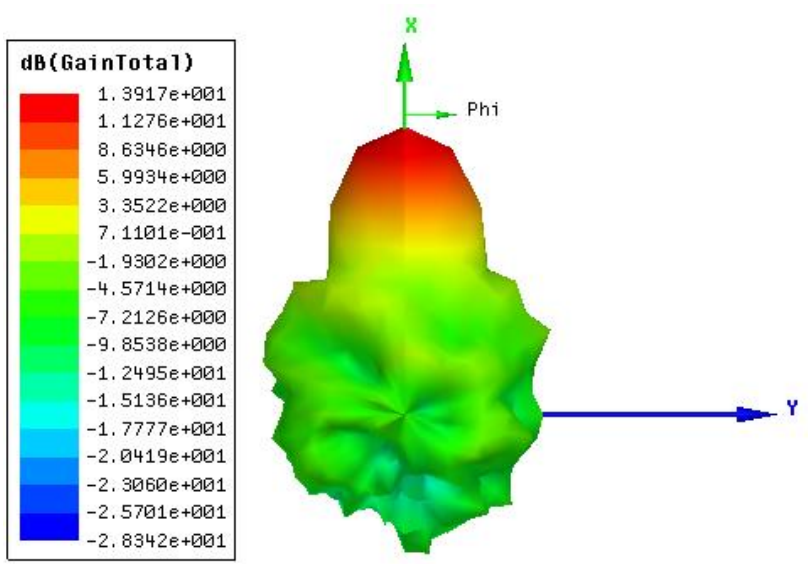

(b)
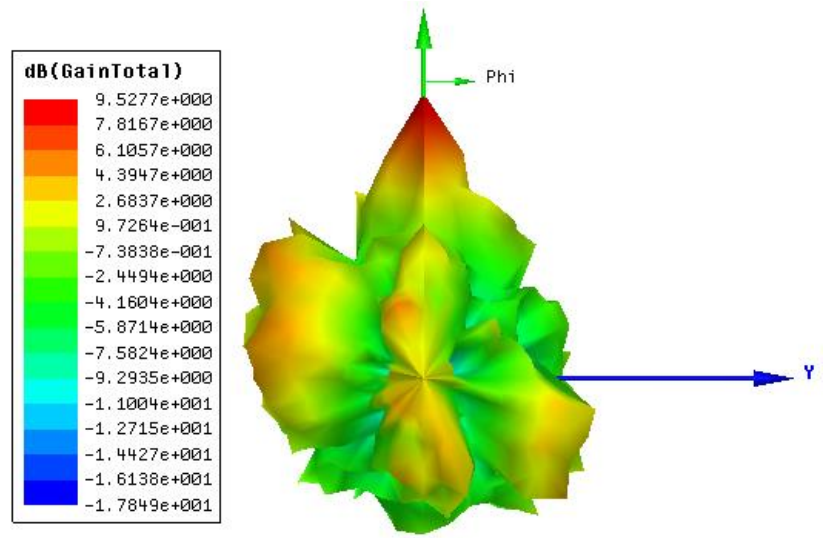

(c)

Figure7. Radiation pattern: (a) at $3.1 \mathrm{GHz}$, (b) at $6.77 \mathrm{GHz},(\mathrm{c})$ at $10 \mathrm{GHz}$.

The recognized gain variation with the frequency of the designed DSVA and the DSVA with the lens is presented in Fig.8. It is observed that owing to the extension of the substrate to compose a half elliptic lens the gain enhanced significantly within the operating frequency band.

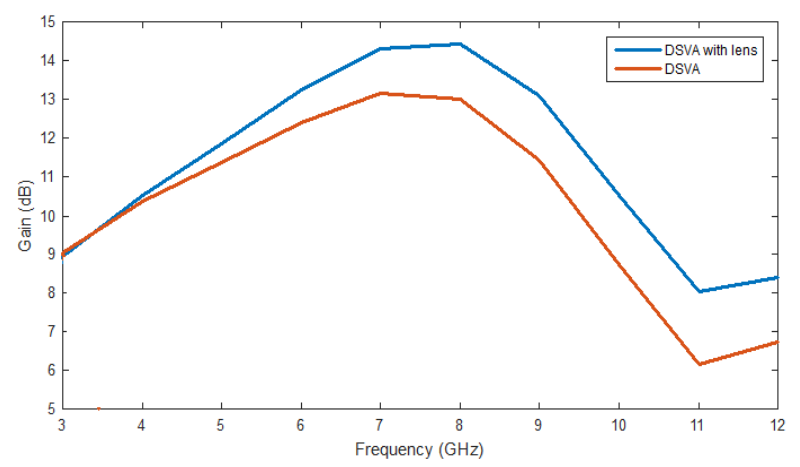

Figure8. Gain variation with the frequency of DSVA with the lens.

\subsection{Simulation and Measurement Results of the DSVA}

In order to support the antenna performance, the DSVA is fabricated and then measured by utilizing a vector network analyzer as presented in Fig.9. From this test, it is found a good match between the measured and simulated results as presented in Fig.10. 


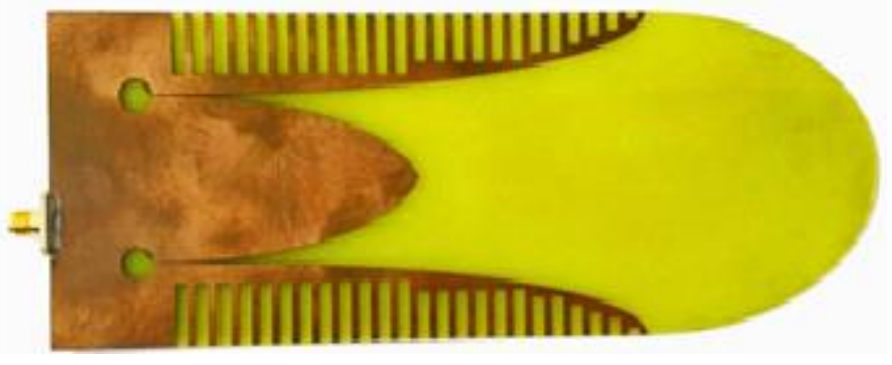

Figure9. The DSVA practical manufacturing configuration

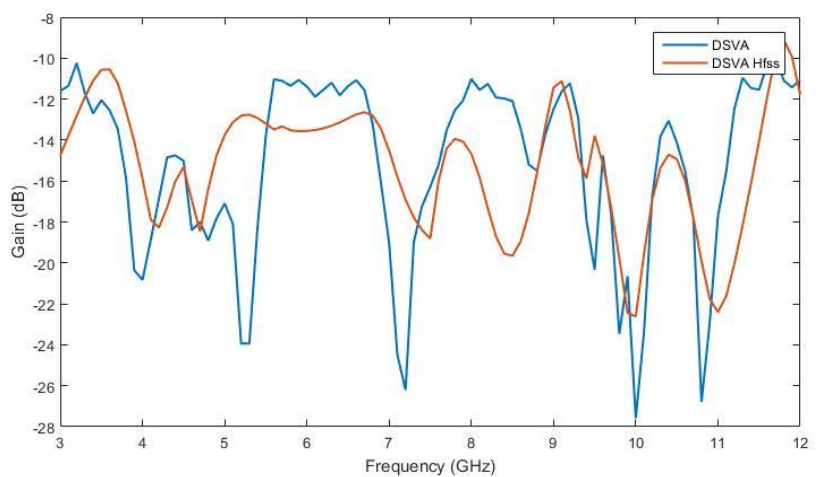

Figure10. The measured and simulated results of DSVA

It can be noticed that the measurement results of the DSVA structure presented a good agreement with a bit different compared with the simulation results where this difference is due to some flaws that may happen over the fabrication process and the environment of testing.

\section{Conclusions}

In this paper, a dual-slot antenna with slots and a dielectric lens is designed. an E-field distribution is created at the aperture of the dualslot construction appear like a standing wave, as a conclusion, the gain is enhanced. The designed DSVA has a UWB frequency domain from (3) $\mathrm{GHz}$ up to (10) GHz. Radiation patterns and the input of the proposed DSVA have been simulated to recognized the designed DSVA executing principles, thus the gain of the designed DSVA reaches $(14.5 \mathrm{~dB})$ which is very suitable for imaging applications.

\section{Conflict of Interest}

The publication of this article causes no conflict of interest.

\section{References}

1. F. Zhang,G.Y.Fang, Y. C. Ji,H. J. Ju, and J. J.Shao,"Anovel compact double exponentially tapered slot antenna (DETSA) for GPR applications," IEEE Antennas Wireless Propag. Lett. vol. 10, pp. 195-198, 2011.

2. J. Bourqui, M. Okoniewski, and E. C. Fear, "Balanced antipodal Vivaldi antenna with dielectric director for near-field microwave imaging," IEEE Trans. Antennas Propag. vol. 58, no. 7, pp. 2318-2326, Jul. 2010.

3. E. W. Reid, L. Ortiz-Balbuena, A. Ghadiri, and K. Moez, "A 324- element Vivaldi antenna array for radio astronomy instrumentation," IEEE Trans. Antennas Propag. vol. 61, no. 1, pp. 241-249, Jan. 2012.

4. Abedian, M., Rahim, S.K.A., Danesh, S., Hakimi, S., and Cheong, L.Y.: 'Novel design of compact UWB dielectric resonator antenna with dual-band-rejection characteristics for WiMAX/WLAN bands', Microw. Wirel. Compon. Lett., 2015, 14, pp. 245-248

5. Islam, M.M., Islam, M.T., Samsuzzaman, M., and Faruque, M.R.I.: 'Compact metamaterial antenna for UWB applications', Electron. Lett. 2015, 51, (16), pp. 12221224.

6. A. Elsherbini ET. al., "UWB antipodal Vivaldi antennas with protruded dielectric rods for higher gain, symmetric patterns and minimal phase center variations," IEEE International Symposium on Antennas and Propagation Society, pp. 1973-1976, jun. 2007.

7. A. Dhouibi, S. N. Burokur, A. LustracA. Priou, "X-band metamaterial based Luneburg 
lens antenna," IEEEE International Symposium on Antennas and Propagation Society, pp. 1292-1293, Jul. 2013.

8. J. Shin, D. H. Schaubert, "A parameter study of strip line-fed Vivaldi notch-antenna arrays," IEEE Trans. Antennas. Propag, vol. 47, no. 5, pp. 879-886, May 1999.

9. Walter, E., Ortiz-Balbuena, L., Ghadiri, A., and Moez, K.: 'A 324-element Vivaldi antenna array for radio astronomy instrumentation', IEEE Trans. Antennas Propag., 2012, 61, (1), pp. 241-249

10. E. W. Reid, L. Ortiz-Balbuena, A. Ghadiri, and K. Moez, "A 324- element Vivaldi antenna array for radio astronomy instrumentation," IEEE Trans. Antennas Propag., vol. 61, no. 1, pp. 241-249, Jan. 2012.

11. T. J. Ellis and G. M. Rebeiz, "MM-wave tapered slot antennas on micro machined photonic bandgap dielectrics," in IEEE MTT-S Int. Microw. Symp. Dig., 1996, pp. 1157-1160.

12. T. G. Lim, H. N. Ang, I. D. Robertson, and B. L. Weiss, "Integrated millimeter-wave tapered slot antenna using conductor strip gratings," Microw. Antennas Propag., vol. 4, no. 9, pp. 1216-1223, Apr. 2010.

13. A. Elsherbini, C. Zhang, S. Lin, and M. Kuhn, "UWB antipodal Vivaldi antennas with protruded dielectric rods for higher gain, symmetric patterns and minimal phase center variations," in Proc. IEEE Antennas Propag. Soc. Int. Symp., 2007, pp. 1973-1976.

14. B. Zhou and T. J. Cui, "Directivity enhancement to Vivaldi antennas using compactly anisotropic zero-index metamaterials," IEEE Antennas Wireless Propag. Lett. vol. 10, pp. 326-329, 2011.

15. Ellis, T.J., and Rebeiz, G.M.: 'MM-wave tapered slot antennas on micro machined photonic band gap dielectrics'. IEEE MTTS Int. Microwave Symp. Digest, San
Francisco, CA, USA, June 1996, pp. 11571160

16. Wang, Y.W. Wang, G.M., Gao, X.J., and Zhou, C.: 'Double-slot Vivaldi antenna with improved gain', Electron. Lett., 2013, 49, (18), pp. 1119-1121

17. Ansoft high frequency structure simulator (HFSS). [Online] Available: www.ansys.com 